\title{
Avaliação do Sistema de Informações Hospitalares do SUS no Tratamento Cirúrgico da ATM: Um Estudo de 10 Anos
}

\author{
Evaluation of the SUS Hospital Information System in Surgical Treatment of the \\ TMJ: A 10-Year Study
}

\author{
João Mykael Alves Xavier ${ }^{1}$ \\ Myllena Alves Xavier ${ }^{2}$ \\ Igor Figueiredo Pereira ${ }^{3}$ \\ Susane de Farias Gomes ${ }^{4}$ \\ Renata de Souza Coelho Soares ${ }^{5}$ \\ Nadja Maria da Silva Oliveira ${ }^{5}$ \\ Kátia Elizabete Galdino ${ }^{6}$ \\ Ana Isabella Arruda Meira Ribeiro ${ }^{5}$
}

\section{RESUMO}

Objetivo: Analisar dados da autorização de internação hospitalar (AlH) consolidados no Sistema de Informações Hospitalares do Sistema Único de Saúde (SIH-SUS), referentes ao tratamento cirúrgico da ATM, em um período de dez anos (2009-2019). Metodologia: Utilizou-se o registro das AlHs no SIH-SUS processadas por meio do DATASUS e a amostra foi composta pelas internações aprovadas em decorrência da realização de procedimentos cirúrgicos da ATM no período em estudo. Para análise estatística foram empregados os testes T- Student e a ANOVA, com nível de significância fixado em $p<$ 0,05 . Resultados: Foram autorizadas 5.458 internações e, destas, a artroplastia foi o procedimento mais executado $(58,04 \%)$ $(p<0,001)$ e a redução cruenta o menos realizado $(3,46 \%)$ $(p<0,001)$. Observou-se que o Sudeste brasileiro $(45,74 \%)$ foi a região na qual teve maior realização dessas cirurgias $(p<0,001)$, sendo o caráter de atendimento eletivo $(59,9 \%)$ o mais predominante e o tratamento cirúrgico de anquilose demandou mais dias de internação $(p=0,015)$. Pode-se verificar na avaliação financeira, que a artroplastia necessitou de mais recursos para os serviços profissionais $(50,33 \%)(p=0,043)$ e o tratamento de cirúrgico de anquilose apresentou, descritivamente, um maior custeio de serviços hospitalares $(53,79 \%)$. Conclusão: O tratamento cirúrgico de ATM assumiu características epidemiológicas heterogêneas no período estudado. Os achados do presente artigo apresentaram informações em vigilância epidemiológica relacionadas às AlHs para o tratamento cirúrgico da ATM, que poderão nortear a tomada de decisão por gestores em saúde pública quanto à compreensão e a alocação de recursos baseada em evidências.

\section{DESCRITORES}

Avaliação de Tecnologia Biomédica. Articulação Temporomandibular. Cirurgia Maxilofacial. Sistema de Informação Hospitalar. Epidemiologia.

\begin{abstract}
Objective: To analyze data from the Hospitalization Authorization $(\mathrm{AlH})$ consolidated in the Hospital Information System of the Unified Health System (SIH-SUS) for the surgical treatment of TMJ, over a period of ten years (2009-2019). Methodology: The registration of AlHs at SIH-SUS processed through DATASUS was used and the sample was composed of admissions approved as a result of performing TMJ surgical procedures during the study period. For statistical analysis, the T-Student and ANOVA tests were used (significance level set at: $p$ <0.05). Results: 5.458 hospitalizations were authorized, and of these, Arthroplasty was the most performed procedure $(58.04 \%)(p<0.001)$ and Raw Reduction the least performed $(3.46 \%)(p<0.001)$. It was observed that the Southeast of Brazil $(45.74 \%)$ was the region that had the greatest performance of these surgeries $(p<0.001)$, with the character of elective care $(59.9 \%)$ being the most prevalent, and the Surgical Treatment of Ankylosis demanding more days of hospitalization ( $p=$ 0.015 ). It was possible to verify in the financial evaluation, that Arthroplasty needed more resources for professional services $(50.33 \%)(p=0.043)$ and the Surgical Treatment of Ankylosis presented, descriptively, a higher cost of hospital services (53, 79\%). Conclusion: Surgical treatment of TMJ assumed heterogeneous epidemiological characteristics in the studied period. The findings of the present article presented information on epidemiological surveillance related to AlHs for the surgical treatment of TMJ, which may guide decision making by public health managers regarding the understanding and allocation of evidence-based resources.
\end{abstract}

\section{DESCRIPTORS}

Technology Assessment Biomedical. Joint Temporomandibular. Surgery Maxillofacial. Hospital Information Systems. Epidemiology.

${ }^{1}$ Graduando em Odontologia, Universidade Estadual da Paraíba, Campina Grande, Paraíba, Brasil.

${ }^{2}$ Cirurgiã-Dentista, Universidade Estadual da Paraíba. Especialista em Ortodontia e Mestre em Ciência e Tecnologia em Saúde, Campina Grande, Paraiba, Brasil.

${ }^{3}$ Professor Mestre do Departamento de Odontologia da Universidade Estadual da Paraíba, Campina Grande, Paraíba, Brasil.

${ }^{4}$ Professora Mestre do Departamento de Engenharia de Produção da Universidade Federal de Alagoas, Arapiraca/Penedo, Alagoas, Brasil. ${ }^{5}$ Professora Doutora do Departamento de Odontologia da Universidade Estadual da Paraíba e Docente Permanente do Mestrado Profissional em Ciência e Tecnologia em Saúde da Universidade Estadual da Paraíba, Campina Grande, Paraíba, Brasil.

${ }^{6}$ Professora Doutora do Departamento de Computação da Universidade Estadual da Paraíba e Docente Permanente do Mestrado Profissional em Ciência e Tecnologia em Saúde da Universidade Estadual da Paraíba, Campina Grande, Paraíba, Brasil. 
$\mathrm{O}^{\mathrm{t}}$ tratamento cirúrgico da articulação temporomandibular (ATM) possui indicações para deformidades congênitas ou adquiridas nas estruturas desse plexo anatômico, instituído para casos de inflamação/infecção, principalmente, relacionados ao disco articular, anquilose e luxações recidivantes ${ }^{1}$. A união fibro-óssea entre o côndilo mandibular e a cavidade glenóide pode estar presente em todas as faixas etárias, o procedimento denominado artroplastia tem por objetivo sanar essas condições através da reconstrução articular².

As desordens articulares podem ser consideradas como sintomas indicativos à realização de cirurgia da ATM quando não se obtêm sucesso nas terapias conservadoras. Nessa perspectiva, a requisição de exames imaginológicos e a coleta do relato dos pacientes devem ser avaliadas com cautela ${ }^{3}$. As patologias que acometem a ATM de maneira expressiva influenciam na função mastigatória, movimentos articulares, limitação de abertura bucal e estética. Tais fatores podem acarretar consequências psicológicas aos indivíduos, o que pode prejudicar a sua qualidade de vida nos âmbitos social e profissional ${ }^{4}$.

Ao traçar o perfil de pesquisas financiadas por editais do SUS no Distrito Federal do Brasil, foi evidenciada a necessidade de propostas que priorizem avaliações no sistema, por meio de Políticas de Ciência, Tecnologia e Inovação em Saúde 5 . Nota-se a presença de um ambiente favorável à implementação de novos conhecimentos nos processos avaliativos em saúde, visto que a própria legislação promulga a realização de audiências públicas para tomar essas decisões na Comissão Nacional de Avaliação de Tecnologias no SUS (CONITEC) ${ }^{6}$.

É importante pontuar que existe uma necessidade quanto à realização de análises financeiras sobre as despesas e alterações no setor de saúde, as quais garantam, por meio de pesquisas, informações neste âmbito. Estas devem ser disseminadas a gestores e a sociedade civil em busca da implementação de adequadas políticas públicas de custeamento que garantam os princípios básicos do SUS ${ }^{7}$. Em 1991, foi implementado no Brasil, o Departamento de Informática do Sistema Único de Saúde (DATASUS) como provedor de soluções em softwares para as secretarias estaduais e municipais de saúde ${ }^{8}$. Apesar do sistema oferecer dados epidemiológicos, existe uma deficiência quanto a ampliação das informações sobre as condições de saúde da população brasileira ${ }^{9}$.

Nesse contexto, o presente estudo buscou averiguar os sistemas de informações hospitalares do SUS no tratamento cirúrgico da ATM, em um período de dez anos, tendo como objetivo descrever as informações obtidas pelo DATASUS, a partir de uma análise epidemiológica pautada na avaliação da usabilidade de dados informatizados para saúde.

\section{METODOLOGIA}

Trata-se de um estudo ecológico com abordagem indutiva, procedimento comparativo-descritivo e técnica de documentação indireta ${ }^{10}$. Utilizaram-se dados de acesso aberto e domínio público obtidos no Sistema de Informações Hospitalares do SUS (SIH-SUS) processados no Portal do Departamento de Informática do Sistema Único de Saúde (DATASUS) $)^{8}$.

A amostra foi composta pelos pacien- 
tes que tiveram internações aprovadas em decorrência da realização de procedimentos cirúrgicos da ATM na atenção terciária do SUS. Foram incluídas todas as AlHs, quanto aos procedimentos cirúrgicos executados para tratamento da ATM, envolvendo indivíduos de todos gêneros e faixas etárias cadastrados em hospitais atendidos pelo Sistema Único de Saúde no período de janeiro 2010 a janeiro 2020. Foram excluídas informações sobre grupos e subgrupos de procedimentos, gestão pública, esfera/ natureza jurídica e regra contratual, decorrentes da realização dos procedimentos.

Para coleta de dados, três pesquisadores previamente calibrados consultaram de maneira independente a plataforma DATASUS $^{8}$ (link < http://www2.datasus.gov.br/ DATASUS/index.php>). Ao acessar a página inicial, selecionou-se o ícone "Informações de saúde (TABNET)" e, em seguida, escolheu-se a opção "Assistência à saúde", a fim de consultar os dados sobre a "Produção Hospitalar (SIH/SUS)".

Na plataforma seguinte, foram aplicados os filtros que geraram informações em forma de tabelas compostas por linha, coluna, conteúdo e período disponível. Assim, foram selecionadas as seguintes informações: $\mathrm{AlH}$ aprovadas, Tipo de procedimento (artroplastia da articulação temporomandibular-redicivante ou não, tratamento cirúrgico de anquilose da articulação temporomandibular e redução cruenta de luxação da articulação temporomandibular-redicivante ou não); Caráter de atendimento (urgência, eletivo, outros); Região do Brasil; Ano de processamento (20102020); Valor dos serviços hospitalares; Valor dos serviços profissionais. Cada uma destas variáveis compuseram os dados referentes às características da internação dos pacientes atendidos no período em estudo.

Realizou-se a análise estatística descritiva objetivando caracterizar a amostra, tendo sido calculadas as frequências absolutas e os percentuais para as variáveis categóricas, bem como, as medidas de tendência central e de variabilidade para as variáveis quantitativas. Empregaram-se os testes de Kolmogrov-Smirnov (para verificar a normalidade dos dados), como também, os T-Student e ANOVA (para análises inferenciais), através do software SPSS versão 20.0, com um nível de significância fixado em $p<0,05$.

\section{RESULTADOS}

Ao analisar as médias de internação, referentes aos diferentes procedimentos executados ao longo dos anos, observou-se que a artroplastia de ATM foi a cirurgia mais realizada $(58,04 \%)$, seguido do tratamento cirúrgico de anquilose de ATM (38,50\%), enquanto a cirurgia de redução cruenta da ATM foi a menos executada $(3,46 \%)(p<0,001)$, o que pode ser observado na Tabela 1. É importante salientar que, pelo número reduzido de internações para realização de redução cruenta de luxação da ATM, esta foi excluída em análises inferenciais posteriores.

Quando verificada a frequência geral de internações aprovadas para a execução dos procedimentos cirúrgicos da ATM em todas as regiões brasileiras, computou-se um total de 5.458 AlH's. Deste número, constatou-se que o Sudeste do Brasil (45,74\%) foi a região onde ocorreram mais atendimentos $(p<0,001)$ (Tabela 2). 
Tabela 1. Estatística referente às internações aprovadas para realização dos procedimentos cirúrgicos da ATM no período em estudo (2010-2020)

\begin{tabular}{c|c|c|c|c|c}
\hline Procedimento & $(\mathrm{n})$ & Porcentagem & Média/Ano & p-valor & Erro Padrão \\
\hline Artroplastia da ATM & 3.168 & $58,04 \%$ & 63,34 &, 000 & 39,75 \\
\hline $\begin{array}{c}\text { Tratamento cirúrgico de Anquilose da } \\
\text { ATM }\end{array}$ & 2.101 & $38,50 \%$ & 41,64 &, 000 & 40,0 \\
\hline $\begin{array}{c}\text { Redução Cruenta de Luxação da } \\
\text { ATM }\end{array}$ & 189 & $3,46 \%$ & 3,78 &, 000 & 40,4 \\
\hline
\end{tabular}

* Dados seguiram normalidade pelo Teste de Kolmogrov-Smirnova , p-valor > 0,05.

*Test-T para amostras pareadas mostrando a diferença entre as médias dos procedimentos, p-valor $<0,05$. Fonte: Pesquisa Direta. (2020)

Foi avaliada a permanência hospitalar no período e as regiões em estudo, de acordo com cada procedimento, demonstrando que o tratamento de anquilose da ATM foi a cirurgia que demandou mais tempo de internação hospitalar $(p=0,015)(E P= \pm 0,38)$, o qual apresentou, em média, aproximadamente 4 dias de internação em suas execuções (Gráfico 1).

Averiguou-se, dentre todos os procedimentos executados no Brasil, no período em estudo, que o caráter de atendimento eletivo $(59,9 \%)$ apresentou maior prevalência que a urgência $(38,5 \%)$ e os outros tipos de caráter de atendimento $(1,6 \%)$ ("acidente no local ou trajeto de trabalho", "outros tipos de acidente de trânsito", "outros tipos de lesão por envenenamento por agentes químicos e físicos") (Gráfico 2).

$\mathrm{Na}$ análise financeira dos serviços profissionais foram gastos um total de $\mathrm{R} \$$ 944.088,97, nos últimos dez anos. Inferiu-se que a artroplastia da ATM foi o procedimento que demandou mais recursos $(50,33 \%)$, em média, foram gastos $\mathrm{R} \$ 95.036,14$ por região na realização dessa cirurgia $(p=0,043)(T a-$ bela 3).

Quando foram analisadas as finan- ças referentes aos serviços hospitalares, observou-se um total $1.856 .919,89$ gastos nos últimos dez anos. Diante desse valor, constatou-se de forma descritiva que o tratamento cirúrgico de anquilose da ATM foi o procedimento que demandou mais recursos $(43,75 \%)$, tendo sido utilizados, em média, R\$198.372,75 por região, todavia, os valores gastos de acordo com cada procedimento, não diferiram estaticamente ( $p=0,052)$.

\section{DISCUSSÃO}

Dados informatizados fortalecem de maneira substancial a capacidade de pesquisadores para análise, coleta e disseminação do conhecimento obtido. A utilização desses recursos maximiza o uso efetivo de informações das instituições de saúde no atendimento à população e a compreensão de vigilância de doenças, que por sua vez, dão consistência às tomadas de decisões em tempo real ${ }^{12}$.

A presente pesquisa apontou a artroplastia da ATM como a cirurgia que teve mais solicitações no período em estudo (58,04\%; $p<0,001)$. Entretanto, a interpretação desse 
Tabela 2. Relação entre as médias da execução de procedimentos cirúrgicos da ATM nas regiões do Brasil, entre 2010 a 2020

\begin{tabular}{|c|c|c|c|c|c|}
\hline Procedimento & Região Analisada & $\begin{array}{l}\text { Regiões } \\
\text { Brasileiras }\end{array}$ & Diferença Média & $\mathrm{p}$-valor & $\begin{array}{c}\text { Erro } \\
\text { Padrão }\end{array}$ \\
\hline \multirow[t]{5}{*}{ Artroplastia da ATM } & Norte & $\begin{array}{l}\text { Nordeste } \\
\text { Sudeste } \\
\text { Sul } \\
\text { Centro-oeste }\end{array}$ & $\begin{array}{c}-38,100 \\
-119,000 \\
-41,100 \\
2,000\end{array}$ & $\begin{array}{l}, 156 \\
, 000 \\
107 \\
1,000\end{array}$ & 16,379 \\
\hline & Nordeste & $\begin{array}{l}\text { Norte } \\
\text { Sudeste } \\
\text { Sul } \\
\text { Centro-oeste }\end{array}$ & $\begin{array}{c}38,100 \\
-80,900 \\
-3,000 \\
40,100\end{array}$ & $\begin{array}{c}, 156 \\
, 000 \\
1,000 \\
, 121\end{array}$ & 16,379 \\
\hline & Sudeste & $\begin{array}{l}\text { Norte } \\
\text { Nordeste } \\
\text { Sul } \\
\text { Centro-oeste }\end{array}$ & $\begin{array}{c}119,000 \\
80,900 \\
77,900 \\
121,000\end{array}$ & $\begin{array}{l}, 000 \\
, 000 \\
, 000 \\
, 000\end{array}$ & 16,379 \\
\hline & Sul & $\begin{array}{l}\text { Norte } \\
\text { Nordeste } \\
\text { Sudeste } \\
\text { Centro-oeste }\end{array}$ & $\begin{array}{c}41,100 \\
3,000 \\
-77,900 \\
43,100\end{array}$ & $\begin{array}{l}107 \\
1,000 \\
, 000 \\
, 081\end{array}$ & 16,379 \\
\hline & Centro-oeste & $\begin{array}{l}\text { Norte } \\
\text { Nordeste } \\
\text { Sudeste } \\
\text { Sul }\end{array}$ & $\begin{array}{c}-2,000 \\
-40,100 \\
-121,000 \\
-43,100\end{array}$ & $\begin{array}{l}1,000 \\
, 121 \\
, 000 \\
, 081\end{array}$ & 16,379 \\
\hline \multirow[t]{5}{*}{$\begin{array}{l}\text { Tratamento Cirúrgico } \\
\text { de Anquilose da ATM }\end{array}$} & Norte & $\begin{array}{l}\text { Nordeste } \\
\text { Sudeste } \\
\text { Sul } \\
\text { Centro-oeste }\end{array}$ & $\begin{array}{c}-27,000 \\
82,100 \\
-19,400 \\
-, 700\end{array}$ & $\begin{array}{l}, 015 \\
, 000 \\
, 140 \\
1,000 \\
\end{array}$ & 8,154 \\
\hline & Nordeste & $\begin{array}{l}\text { Norte } \\
\text { Sudeste } \\
\text { Sul } \\
\text { Centro-oeste }\end{array}$ & $\begin{array}{c}27,000 \\
-55,100 \\
7,600 \\
26,300\end{array}$ & $\begin{array}{l}, 015 \\
, 000 \\
, 883 \\
, 019\end{array}$ & 8,154 \\
\hline & Sudeste & $\begin{array}{l}\text { Norte } \\
\text { Nordeste } \\
\text { Sul } \\
\text { Centro-oeste }\end{array}$ & $\begin{array}{l}82,100 \\
55,100 \\
62,700 \\
81,400\end{array}$ & $\begin{array}{l}, 000 \\
, 000 \\
, 000 \\
, 000\end{array}$ & 8,154 \\
\hline & Sul & $\begin{array}{l}\text { Norte } \\
\text { Nordeste } \\
\text { Sudeste } \\
\text { Centro-oeste } \\
\end{array}$ & $\begin{array}{c}19,400 \\
-7,600 \\
-62,700 \\
18,700 \\
\end{array}$ & $\begin{array}{l}, 140 \\
, 883 \\
, 000 \\
, 166 \\
\end{array}$ & 8,154 \\
\hline & Centro-oeste & $\begin{array}{l}\text { Norte } \\
\text { Nordeste } \\
\text { Sudeste } \\
\text { Sul }\end{array}$ & $\begin{array}{c}, 700 \\
-26,300 \\
-81,400 \\
-18,700\end{array}$ & $\begin{array}{l}1,000 \\
, 019 \\
, 000 \\
, 166\end{array}$ & 8,154 \\
\hline
\end{tabular}

*Teste ANOVA de um fator apresentando a região Sudeste como a que executou mais procedimentos, $p<0,005$. ${ }^{*} \mathrm{~A}$ realização do teste ANOVA já comprova a normalidade dados por ser um teste estatisticamente robusto. Fonte: Pesquisa Direta. (2020) 
Gráfico 1. Estatística das médias referentes ao tempo de permanência hospitalar para realização de procedimentos cirúrgicos da ATM, entre 2010 a 2020

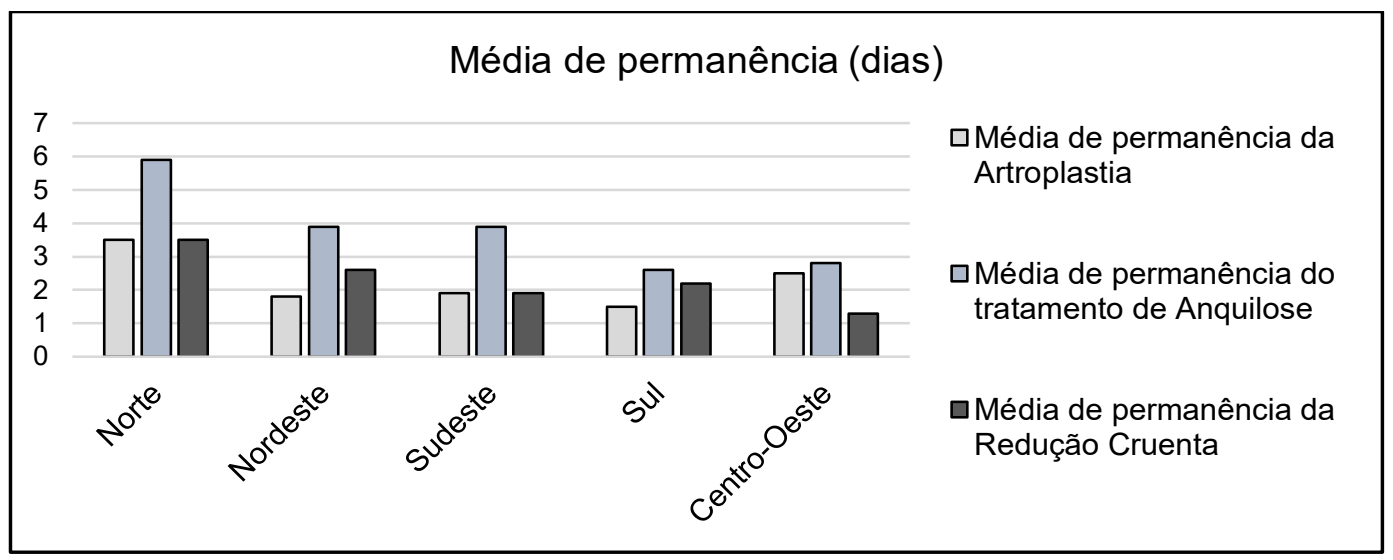

* Dados seguiram normalidade pelo Teste de Kolmogrov-Smirnova , p-valor > 0,05.

*Test-T para amostras pareadas mostrando a diferença entre as médias dos procedimentos, p-valor < 0,05. Fonte: Pesquisa Direta. (2020).

Gráfico 2. Solicitação dos procedimentos cirúrgicos da ATM conforme caráter de atendimento, entre 2010 a 2020

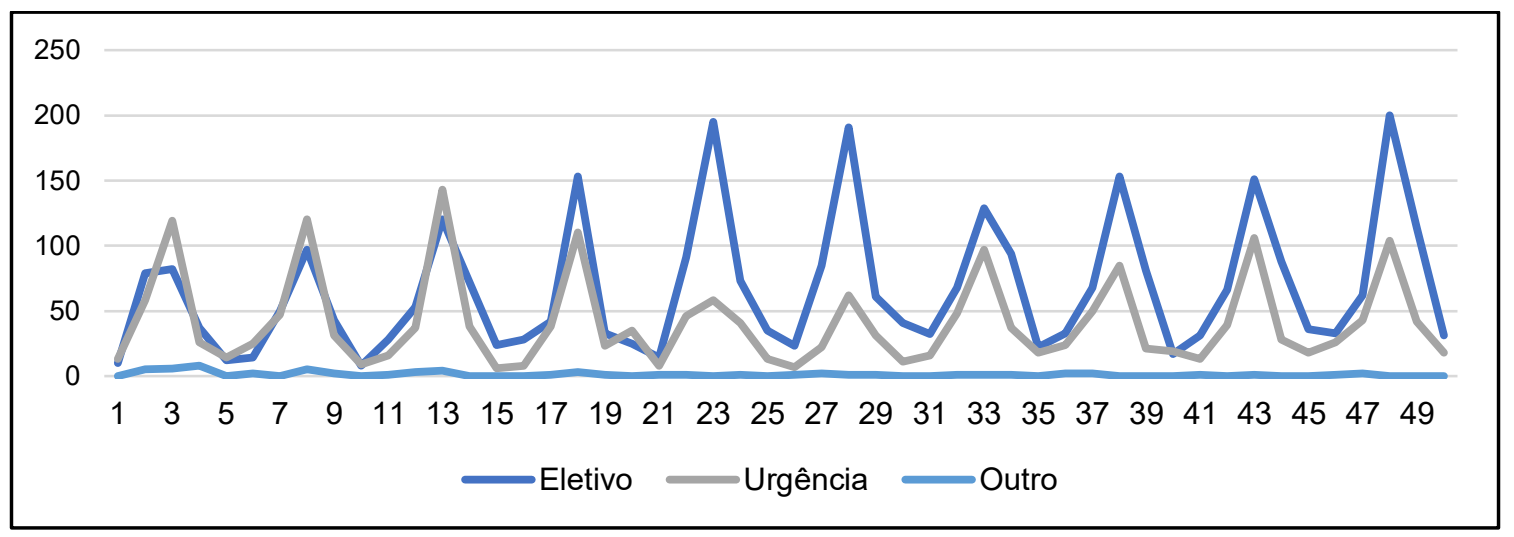

Fonte: Pesquisa Direta. (2020)

Tabela 3. Estatística referente aos gastos financeiros dos serviços profissionais para realização de procedimentos cirúrgicos da ATM no período estudado (2010-2020)

\begin{tabular}{c|c|c|c|c|c}
\hline Procedimento & $(\mathrm{n})$ & Porcentagem & $\begin{array}{c}\text { Média por Re- } \\
\text { gião }\end{array}$ & $\mathrm{p}$-valor & $\begin{array}{c}\text { Erro } \\
\text { Padrão }\end{array}$ \\
\hline Artroplastia da ATM & $\mathrm{R} \$ 475.180,73$ & $50,33 \%$ & $\mathrm{R} \$ 95.034,14$ & 0,043 & 32539,6 \\
\hline $\begin{array}{c}\text { Tratamento cirúrgico } \\
\text { de Anquilose da ATM }\end{array}$ & $\mathrm{R} \$ 445.490,72$ & $47,18 \%$ & $\mathrm{R} \$ 89.096,14$ & 0,048 & 31677,8 \\
\hline $\begin{array}{c}\text { Redução Cruenta de } \\
\text { Luxação da ATM }\end{array}$ & $\mathrm{R} \$ 23.417,52$ & $2,49 \%$ & $\mathrm{R} \$ 4.681,41$ & 0,065 & 1857,5 \\
\hline
\end{tabular}

* Dados seguiram normalidade pelo Teste de Kolmogrov-Smirnova , p-valor > 0,05

*Test-T para amostras pareadas mostrando a diferença entre as médias dos valores dos serviços profissionais entre os procedimentos, p-valor < 0,05. Fonte: Pesquisa Direta. (2020) 
Tabela 4. Estatística aos gastos financeiros dos serviços hospitalares para realização de procedimentos cirúrgicos da ATM no período estudado (2010-2020)

\begin{tabular}{c|c|c|c|c|c}
\hline Procedimento & $(\mathrm{n})$ & Porcentagem & $\begin{array}{c}\text { Média por } \\
\text { Região }\end{array}$ & $\mathrm{p}$-valor & $\begin{array}{c}\text { Erro Pa- } \\
\text { drão }\end{array}$ \\
\hline Artroplastia da ATM & $\mathrm{R} \$ 812.494,23$ & $43,75 \%$ & $\mathrm{R} \$ 162.241,27$ & 0,039 & 53728,89 \\
\hline $\begin{array}{c}\text { Tratamento cirúrgico de } \\
\text { Anquilose da ATM }\end{array}$ & $\mathrm{R} \$ 998.942,94$ & $53,79 \%$ & $\mathrm{R} \$ 198.372,75$ & 0,052 & 72546,78 \\
\hline $\begin{array}{c}\text { Redução Cruenta de } \\
\text { Luxação da ATM }\end{array}$ & $\mathrm{R} \$ 45.482,72$ & $2,44 \%$ & $\mathrm{R} \$ 8.008,69$ & 0,065 & 3581,59 \\
\hline
\end{tabular}

* Dados seguiram normalidade pelo Teste de Kolmogrov-Smirnov ${ }^{a}$, p-valor > 0,05

*Test-T para amostras pareadas mostrando a diferença entre as médias dos valores dos serviços Hospitalares entre os procedimentos, p-valor < 0,05. Fonte: Pesquisa Direta. (2020)

resultado não deve se limitar tão somente aos números de internações, uma vez que o tratamento de cirúrgico dessa comorbidade é solicitado quando ocorre o esgotamento de todas alternativas de terapias conservadoras. Os pacientes que se submetem a esse tipo de procedimento têm um longo histórico de tratamento, tanto na fase pré, como em seu pós-operatório ${ }^{13,14}$. Nessa perspectiva, é importante salientar que existe a necessidade de estudos epidemiológicos, a fim caracterizar aspectos sociodemográficos, qualidade de vida, incidência e prevalência dessa patologia em diferentes grupos populacionais.

O SIH apontou o Sudeste do Brasil como o território com maior realização de procedimentos para o tratamento cirúrgico da ATM. Inicialmente, uma maneira de fundamentar este resultado foi por meio da observação do maior tamanho populacional dessa região em relação às demais ${ }^{15}$. Outra particularidade, foram os avanços por ações do SUS no tratamento de doenças não transmissíveis e a alocação de recursos nessa região, fatos os quais têm sido evidenciados em pesquisas anteriores $^{16,17}$.
O Produto Interno Bruto (PIB) do Brasil, no segundo trimestre do ano de 2020 , obteve um valor de 1,653 trilhão de reais no tocante ao fluxo de novos bens e serviços finais produzidos, entretanto, estes valores compõem uma síntese da macroeconomia, sem expressar fatores importantes como, a distribuição de renda, a qualidade de vida e a saúde ${ }^{18,19}$. Nesse sentido, os resultados da presente pesquisa tiveram o papel de traçar o perfil financeiro do tratamento cirúrgico da articulação temporomandibular, evidenciando de forma descritiva, que a cirurgia de anquilose da ATM $(53,79 \%)$ foi o procedimento mais dispendioso quanto aos serviços hospitalares, bem como, comprovado estatisticamente que a artroplastia de ATM $(50,33 \% ; p=0,053)$ demandou mais recursos quanto aos serviços profissionais, fato este que pôde ser justificado pelo número de execuções desses procedimentos.

$\mathrm{O}$ presente estudo mostrou que o tratamento cirúrgico de anquilose da ATM foi o procedimento que exigiu mais tempo de permanência hospitalar (aproximadamente, 4 
dias de internação), o que pode estar associado à etiologia desse quadro patológico, uma vez que pesquisas apontaram que o trauma é a causa mais comum para o tratamento dessa condição ${ }^{20,21}$. Dessa forma, o paciente que se apresenta ao serviço de saúde com esta comorbidade pode estar acometido com outros traumas associados, necessitando assim, de mais dias de internação. Ademais, uma análise para determinar o tempo médio de internação das vítimas de fraturas faciais submetidos a tratamento cirúrgico em dois hospitais de urgência e emergência da $\mathrm{Pa}$ raíba, apontou uma média de 4,5 dias ${ }^{22}$, valor semelhante aos resultados apresentados no presente estudo.

Uma forma de tentar compreender os fatores causais que levaram os tratamentos eletivos a serem mais solicitados que os de urgência, ocorre por meio do entendimento multifatorial ${ }^{23}$ e multidisciplinar dessas doenças, uma vez que as patologias que acometem a ATM podem ser diagnosticadas e tratadas por diferentes grupos de profissionais ${ }^{24}$ (cirurgiões bucomaxilofacial, neurologistas e fisioterapeutas $)^{25}$, tornando-se assim, mais viável o agendamento dos procedimentos, conforme a demanda e atuação das diferentes especialidades nesses casos, além de demandarem um grande planejamento, tendo em vista que, geralmente, outras terapias conservadoras foram utilizadas sem obtenção de sucesso.

Uma das principais responsabilidades da Vigilância Epidemiológica é informar, integrar e planejar às Redes de Atenção à Saúde a fim de garantir o desenvolvimento de intervenções transdisciplinares e resolutivas para o SUS ${ }^{26}$. Assim, a presente pesquisa apresentou de maneira inédita as características da atenção em saúde aos pacientes que necessitaram de tratamentos cirúrgicos da ATM e buscou compreender seus determinantes sociais, biológicos e econômicos, a fim de planejar intervenções baseadas em evidências cientificas para o manejo dessa morbidade.

\section{CONCLUSÃO}

Este artigo concluiu que o tratamento cirúrgico da ATM no SUS mostrou características heterogêneas no período estudado. Pôde-se notar, dentre a análise das internações aprovadas, que a artroplastia foi o procedimento mais executado e a redução cruenta o menos realizado. O Sudeste brasileiro mostrou-se como a região que teve uma maior realização dessas cirurgias, sendo o caráter de atendimento eletivo o mais predominante. Quanto à permanência hospitalar, foi apontado que o tratamento cirúrgico de anquilose foi o que precisou de mais dias de internação.

Nas análises financeiras de gastos em saúde, pode-se verificar que a artroplastia, demandou mais recursos para os serviços profissionais e o tratamento de cirúrgico de anquilose necessitou de um maior custeio de serviços hospitalares.

Sugere-se que esse estudo apresente-se como uma ferramenta de avaliação em saúde no âmbito da vigilância epidemiológica, visando, dessa forma, ser utilizado para posteriores tomadas de decisões baseadas em evidências as quais apontem as principais características e necessidades dos usuários do SUS para gestores em saúde pública no país. 


\section{REFERÊNCIAS}

1. Bouloux G, Koslin MG, Ness G, Shafer D. Temporomandibular Joint Surgery. J Oral Maxillofac Surg. 2017; 75(8):195-223.

2. Santos LA, Araújo MA, Nogueira RVB. Anquilose na Articulação Temporomandibular: Uma revisão com ênfase em tratamento. Revista da ACBO. 2018; 27(1):48-53.

3. Antonarakis GS, Kalberer N, Courvoisier DS, Scolozzi P. Clinical predictive factors for temporomandibular disorders following combined orthodontic and orthognathic surgical treatment in patients with Class III malocclusion. Cranio. 2017; 35(6):397-404

4. Cordeiro PCF, Quinelato V, Bonato LL, Braune AS, Santos TA, Casado PL. Artroplastia interposicional para tratamento de anquilose da articulação temporomandibular: relato de caso pediátrico. Rev Port Estomatol Med Dent Cir Maxilofac. 2018; 59(1):54-60.

5. Novaes MRCG, Motta ML, Elias FTS, Silva RE, Silva CCG, Baliero VAT et al. Incentivos e desafios relacionados à condução da pesquisa científica, tecnológica e de inovação no âmbito do Sistema Único de Saúde no Distrito Federal, Brasil. Ciênc. saúde coletiva. 2019; 24(6):2211-2220.

6. Brasil. Ministério da Saúde. Lei 12.401 , de 28 de abril de 2011. Altera a Lei no 8.080 , de 18 de setembro de 1990, para dispor sobre a assistência terapêutica e a incorporação de tecnologia no âmbito do Sistema Único de Saúde - SUS. Diário oficial da União 11 abr. 2011.

7. Massuda A, Hone T, Leles FAG, Castro MC, Atun R. The Brazilian health system at crossroads: progress, crisis and resilience. BMJ Glob Health. 2018; 3(4):1-8.

8. Brasil, Ministério da Saúde. Banco de dados do Sistema Único de Saúde-DATASUS. Sistema de informações hospitalares. 2020.

9. Saldanha RF, Bastos RR, Barcellos C. Microdatasus: pacote para download e pré-processamento de microdados do Departamento de Informática do SUS (DATASUS). Cad. Saúde Pública. 2019; 35(9):1-9.

10. Lakatos EM, Marconi MA. Fundamentos da Metodologia Científica. 6a. ed, São Paulo: Atlas, 2009.

11. Brasil. Ministério da Saúde. Conselho Nacional de Saúde. Resolução n. 466, de 12 de dezembro de 2012. Aprova diretrizes e normas regulamentadoras de pesquisas envolvendo seres humanos. Brasília, Diário Oficial da União 12 dez. 2012.
12. Handley K, Boerma T, Victora C, Evans TG. An inflection piont for country health data. Lancet Glob Health. 2015; 3(8):37-38.

13. Henry A, Inverso G, Granquist EJ. Revision temporomandibular joint arthroplasty for the treatment of acquired metal allergy and review of the literature. Int J Oral Maxillofac Surg. 2020; 49(3):356-360.

14. Barbosa MCSA, Kinoshita AMO, Silveira EMV. Biomateriais utilizados na artroplastia parcial ou total da articulação temporomandibular: uma revisão de literatura. Salusvita. 2018; 37(2):389-403.

15. Instituto Brasileiro de Geografia e Estatística (IBGE). Cidades e Estados. 2020.

16. Saltarelli RMF, Prado RR, Monteiro RA, Machado IE, Teixeira BSM, Malta DC. Mortes evitáveis por ações do Sistema Único de Saúde na população da Região Sudeste do Brasil. Ciênc. saúde coletiva. 2019; 24(3):887-898.

17. Santos LM, Francisco JRS, Gonçalves MA. Controle na alocação de recursos na saúde pública: uma análise nas microrregiões do sudeste brasileiro. Administração Pública e Gestão Social. 2016; 8(2):119-129.

18. Instituto Brasileiro de Geografia e Estatística (IBGE). Produto Interno Bruto. 2020.

19. Instituto de Pesquisa Econômica Aplicada (IPEA/ Ipeadata). Macroeconomia. 2020.

20. Movahed R, Mercuri LG. Management of temporomandibular joint ankylosis. Oral Maxillofac Surg Clin North Am. 2015;27(1):27-35.

21. Xia L, An J, He Y, Xiao E, Chen S, Yan Y et al. Association between the clinical features of and types of temporomandibular joint ankylosis based on a modified classification system. Sci Rep. 2019; 9(1):10493.

22. Porto DE, Carreira PFS, Cavalcante JR. Análise do Tempo Médio de Internação em Pacientes com Fraturas Faciais em Hospitais de Urgência e Emergência da Paraíba - PB. Rev. cir. traumatol. buco-maxilo-fac. 2016, 16(4):18-24.

23. Foletti JM, Cheynet F, Graillon N, Guyot L, Chossegros C. Arthroscopie de l'articulation temporo-mandibulaire. Mise au point [TMJ arthroscopy. A review]. Rev Stomatol Chir Maxillofac Chir Orale. 2016;117(4):273-279. 
24. Hoffman D, Puig L. Complications of TMJ surgery. Oral Maxillofac Surg Clin North Am. 2015; 27(1):109-124.

25. Pelicioli M, Myra RS, Florianovicz VC, Batista JS. Tratamento fisioterapêutico nas desordens temporomandibulares. Rev. Dor. 2017; 18(4):355-361.

26. Franco NG, Villardi JWR, Machado JMH, Souza MS, Brito IF, Santorum JA et al. Vigilância em Saúde brasileira: reflexões e contribuição ao debate da $1^{\text {a }}$ Conferência Nacional de Vigilância em Saúde. Ciênc. saúde coletiva. 2017; 22(10):3137-3148.

\section{CORRESPONDÊNCIA}

João Mykael Alves Xavier

Rua Baraúnas, 351 - Universitário, Campina Grande- PB 58429-500

Email: joaomykael99@gmail.com 